\title{
Costes de construcción y consumos de energía en la rehabilitación energética de un edificio de viviendas situado en Madrid (España)
}

\author{
Construction costs and energy consumptions resulting from energy retrofitting in a residential \\ building in Madrid (Spain)
}

Justo García Navarro (Autor Principal y Autor Oficial de Contacto) justo.gnavarro@upm.es

Grupo de Investigación Sostenibilidad en la Construcción

y en la Industria, giSCI. Universidad Politécnica de Madrid. Ciudad Universitaria, s/n. 28040 Madrid (España)

\author{
Marta Valdivieso Rodríguez \\ Grupo de Investigación Sostenibilidad en la Construcción \\ y en la Industria, giSCl. Universidad Politécnica de \\ Madrid, España \\ marta.vlrd@gmail.com
}

\section{María Jesús González Díaz}

Grupo de Investigación Sostenibilidad en la Construcción y en la Industria, giSCI. Universidad Politécnica de Madrid, España

maria.gdiaz@alumnos.upm.es

\author{
Código: 0180 \\ Fecha de Aceptación: 1 de diciembre de 2013.
}

\section{Resumen}

Se trata de un estudio teórico que analiza la relación entre las medidas necesarias para la rehabilitación energética de un edificio residencial construido en Madrid, su coste y la mejora de la calificación energética. El objetivo es desarrollar propuestas y encontrar soluciones para facilitar a promotores y proyectistas la toma de decisiones relacionadas con la eficiencia energética en intervenciones de rehabilitación. En el caso de estudio abordado se han analizado 2 medidas constructivas de tipo pasivo (actuando sobre la envolvente), 13 medidas de tipo activo (sistemas energéticos diferentes), y las combinaciones necesarias entre ellas para lograr diversas calificaciones energéticas. Se han analizado así 26 hipótesis de rehabilitación energética, con sus calificaciones energéticas resultantes, valorando costes y rentabilidad, así como su incidencia en los consumos energéticos y en las emisiones de $\mathrm{CO}_{2}$. $\mathrm{El}$ éxito de la rehabilitación energética de un edificio depende de la selección de las soluciones adoptadas, del coste de la inversión para realizar las obras, y del ahorro de energía conseguido. Asimismo es necesario tener en cuenta otros aspectos no cuantificables como un mayor confort, un mejor aislamiento acústico, la habitabilidad, la salud, la posibilidad de eliminación de situaciones de pobreza energética o el Abstract

This theoretical study analyses the relation between the needed measures for the energy retrofitting of a residential building constructed in Madrid, their cost and the improvement of the energy rating of the dwellings. The aim of this work is to develop proposals and solutions for building's energy retrofitting that help developers and designers in the decision-making process. In this case study 2 passive systems (acting on the envelope), 13 active systems (regarding energy systems), and the needed combinations between all of them, have been taken into account. Consequently, 26 hypothesis of energy retrofitting, with their respective energy rates, have been analyzed. All of them have been assessed not only from a cost-benefit approach, but also considering consumes and $\mathrm{CO} 2$ emissions. This study will allow finding the most suitable cost-effective solutions in each case. Energy retrofitting means an increase in property value but it can't be only considered in economic terms. It is necessary to take into account other unquantifiable aspects as increased comfort, improved sound insulation, livability, health, or the elimination of energy poverty situations.

aumento de valor de la propiedad.

Keywords: Refurbishment; Energy Retrofitting; Energy Rating; Cost-Effectiveness; Sustainability.

\section{Introducción: Estado de la cuestión}

La Unión Europea aprobó en 2008 la comunicación "Dos veces 20 para el 2020. El cambio climático, una oportunidad para Europa", que posteriormente se ha ido desarrollando hasta definir tres objetivos principales para el año 2020: reducir en un $20 \%$ las emisiones de gases de efecto invernadero (GEI), mejorar en un $20 \%$ la eficiencia energética, y conseguir que las energías renovables representen el $20 \%$ del consumo energético de la de UE (Comisión Europea, 2008). Y teniendo en cuenta que el $40 \%$ del consumo final de energía en la UE corresponde a los edificios, se confirma que el sector de la edificación es un elemento clave para lograr estos objetivos. En esta misma línea, la Comisión Europea aprobó en 2010 la Directiva de Eficiencia Energética en Edificios (EPBD) 2010/31/EU (Parlamento Europeo, 2010). Este documento establece los requisitos mínimos de eficiencia energética de los edificios, desarrolla su metodología de cálculo y su certificado. La UE estima que la implementación de la Directiva 2010/31 puede reducir el consumo de energía en un 5-6\%.
En cuanto a España, el estudio "Potencial de ahorro energético y de reducción de emisiones de $\mathrm{CO}_{2}$ del parque residencial existente en España en 2020" (WWF/Adena, 2010) aporta algunos datos de interés: la edificación representa el $26 \%$ del consumo de energía final: un $17 \%$ las viviendas, y un $9 \%$ los edificios del sector terciario. En el caso de las viviendas, un $53 \%$ de un total aproximado de 25 millones se construyó antes de 1979, cuando se aprobó la primera legislación relativa a la eficiencia energética. En los últimos 18 años, el consumo energético de los hogares ha aumentado en aproximadamente un $50 \%$. Estas cifras confirman la importancia de la rehabilitación energética del parque de viviendas existentes para lograr los objetivos europeos enunciados. El mismo estudio antes citado estima que el sector residencial español tiene capacidad técnica y económica para reducir al menos un $30 \%$ el consumo de energía en las viviendas existentes en 2020 , si bien tal esfuerzo requeriría la renovación de entre medio millón y un millón de hogares al año, aproximadamente el 2,4\% de las viviendas. 


\section{Descripción del problema}

En el contexto descrito, el presente trabajo examina la relación entre las soluciones técnicas rentables que permiten la rehabilitación energética de un edificio residencial, la calificación energética que resulta de la aplicación de estas soluciones, su reducción del consumo y su coste. También establece una evaluación comparativa que permitirá a promotores y proyectistas obtener conclusiones para ser guiados en el proceso de toma de decisiones en proyectos de rehabilitación, buscando las mejores soluciones para cada caso desde los puntos de vista ambiental y económico. Asimismo, este trabajo es continuación de otros anteriores desarrollados por el mismo equipo investigador (García Navarro et al. 2010), que han abordado con planteamientos similares una problemática idéntica en edificios de nueva construcción (García Navarro et al. 2014). La realidad del mercado inmobiliario y la necesidad comúnmente aceptada de que el sector inmobiliario europeo dirija su actividad a la rehabilitación del parque construido existente, han avalado el interés de esta nueva fase denominada precost\&e rehabilitación.

\section{Metodología}

El procedimiento establecido para abordar el caso de estudio ha seguido los siguientes pasos: El análisis del edificio-caso de estudio, considerando sus características constructivas y energéticas, los consumos medios de gas natural, electricidad y agua; y los datos climáticos conforme a las cartas bioclimáticas de Olgyay y Givoni. Asimismo se ha verificado el cumplimiento de la normativa vigente de eficiencia energética, y se ha calculado la calificación energética del edificio con el programa Calener VyP, que es el programa oficial para establecer la calificación energética de viviendas y pequeños edificios del sector terciario en España.

La descripción, catalogación y evaluación de medidas para la mejora de la envolvente del edificio, con la descripción de las estrategias para reducir la demanda de energía y su definición técnica. La descripción, catalogación y evaluación de medidas para la mejora de los sistemas energéticos del edificio. La definición, mediante la combinación de medidas de mejora de la envolvente (pasivas) y de los sistemas energéticos (medidas activas), de 26 hipótesis de rehabilitación. La obtención de resultados sobre cada una de las 26 hipótesis de rehabilitación: calificación energética resultante en función de emisiones de $\mathrm{CO}_{2}\left(\mathrm{kgCO}_{2} / \mathrm{m}^{2} \mathrm{año}\right)$, demanda de energía $\left(\mathrm{kWh} / \mathrm{m}^{2}\right.$ año), consumo y ahorro de energía $\left(\mathrm{kWh} / \mathrm{m}^{2}\right.$ año), coste de construcción $\left(€ / \mathrm{m}^{2}\right)$ y ahorro económico previsto en el consumo de energía ( $€ / \mathrm{m}^{2}$ año). Finalmente, el establecimiento de conclusiones generales sobre la rehabilitación energética, resultados económicos y observaciones acerca de las herramientas utilizadas.

\section{Caso de Estudio}

El caso de estudio es un edificio de 95 apartamentos construido en el Paseo Alameda 48, en Madrid (España) (Figura 1). Se trata de un edificio de propiedad única, que está siendo explotado en alquiler. El edificio es exento, de dimensiones $10,80 \mathrm{~m}$ y $126,11 \mathrm{~m}$, y se estructura en cinco portales (cinco bloques adosados), cada uno con un núcleo de comunicación vertical (Figura 2). Su superficie es de 9.317,60 $\mathrm{m}^{2}$ sobre rasante. De los 95 apartamentos, 65 son de 2 dormitorios y 30 de 3 dormitorios. Sus superficies están comprendidas entre 65,31 y $71,05 \mathrm{~m}^{2}$, en el caso de los apartamentos de 2 dormitorios, y alrededor de los $80,20 \mathrm{~m}^{2}$ los de 3 dormitorios. El edificio tiene orientadas sus fachadas longitudinales hacia Este y Oeste. Se proyectó en 2003 y se terminó de construir en 2005. Está habitado, por lo que se ha considerado que las soluciones adoptadas en la hipotética rehabilitación deben minimizar las molestias a los usuarios.

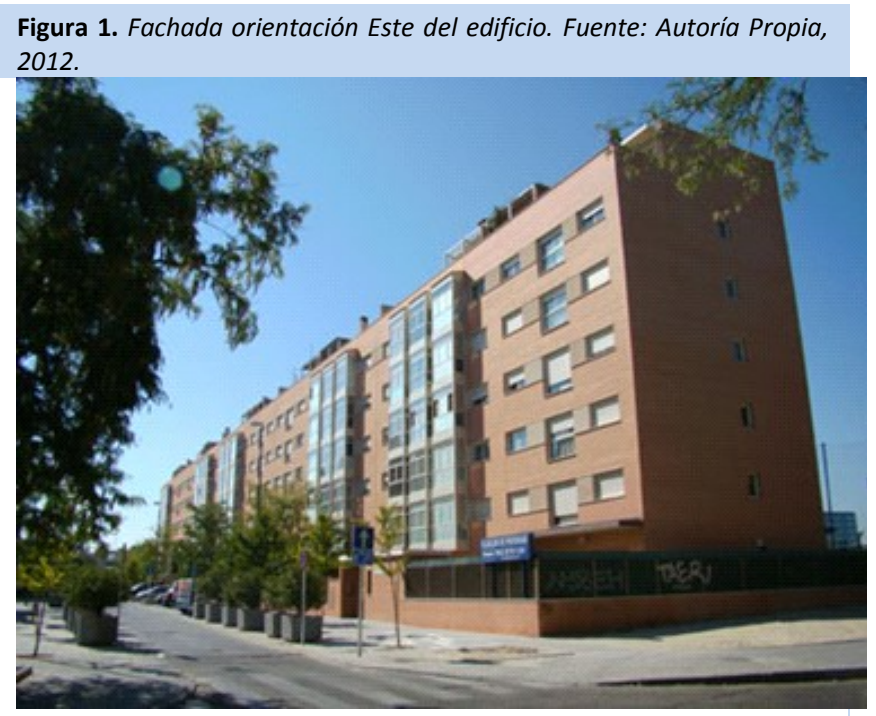

Figura 2. Planta tipo de uno de los bloques. Fuente: TESTA INMOBILIARIA, 2003.

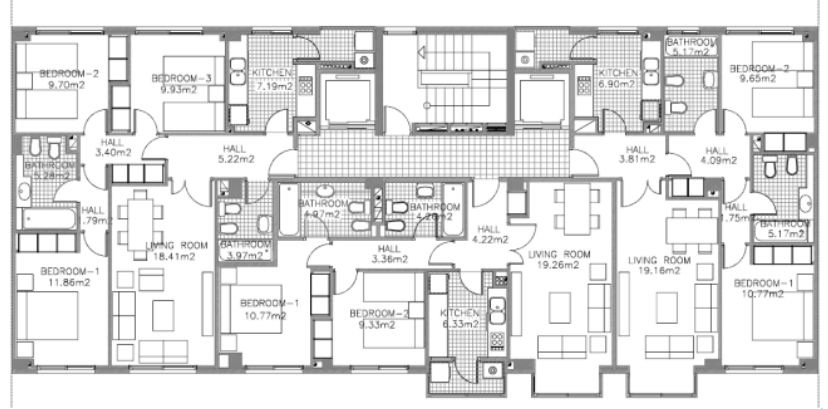

\section{La envolvente del edificio}

El edificio fue construido anteriormente a la entrada en vigor de la normativa "Documento Básico de Ahorro de Energía: Limitación de la demanda energética $\mathrm{DB} H \mathrm{HE}^{\prime}$, que es la aplicación de la directiva EPBD en España, por lo que no la cumple. Los valores límite de transmitancia de los cerramientos de acuerdo con la normativa CTE-DB-HE para el clima de Madrid, en comparación con los que el edificio posee, son los siguientes (Tabla 1$)^{1}$ :

\section{Los sistemas energéticos}

Las viviendas tienen agua caliente sanitaria (ACS) y sistema de calefacción, que se resuelve mediante una caldera convencional de capacidad nominal 27,6 kW, con un rendimiento energético de hasta el $91 \%$; un acumulador de 43 I

\footnotetext{
1 El DB HE-1 ha sido recientemente modificado (septiembre de 2013) y ya no especifica valores límite medios, que son los que se indican en la tabla 1.
} 
integrado en la caldera, y radiadores de aluminio. No tienen refrigeración ni instalaciones de energías renovables.

Tabla 1. Transmitancias de la envolvente. Fuente: elaboración propia 2013.

\begin{tabular}{|c|c|c|c|}
\hline $\begin{array}{l}\text { Elemento de } \\
\text { construcción }\end{array}$ & Descripción & $\begin{array}{l}\text { U Edificio } \\
\text { existente } \\
\left(\mathrm{W} / \mathrm{m}^{2} \mathrm{~K}\right)\end{array}$ & $\begin{array}{l}\text { U Límite DB } \\
\text { HE-1 } \\
\left(\mathrm{W} / \mathrm{m}^{2} \mathrm{~K}\right)\end{array}$ \\
\hline Cubierta & $\begin{array}{l}\text { Cubierta } \\
\text { invertida (40 } \\
\text { mm XPS) }\end{array}$ & 0,56 & 0,38 \\
\hline Fachada & $\begin{array}{l}\text { Fachada doble } \\
\text { (PUR } 40 \mathrm{~mm} \text { ) }\end{array}$ & 0,55 & 0,66 \\
\hline $\begin{array}{l}\text { Cerramiento } \\
\text { en contacto } \\
\text { con el terreno }\end{array}$ & $\begin{array}{l}\text { Muro de } \\
\text { hormigón de } 30 \\
\mathrm{~cm} / \text { Partición } \\
\text { de } 12 \mathrm{~cm}\end{array}$ & $2,82 / 2,70$ & 0,66 \\
\hline \multirow[t]{2}{*}{ Ventanas } & $\begin{array}{l}\text { Marco de la } \\
\text { ventana de } \\
\text { aluminio sin } \\
\text { rotura de } \\
\text { puente térmico }\end{array}$ & 5,70 & \multirow{2}{*}{$\begin{array}{l}2.2 \text { (Norte); } \\
2.6 \text { (Este- } \\
\text { Oeste); } 3.4 \\
\text { (Sur) }\end{array}$} \\
\hline & $\begin{array}{l}\text { Vidrio } 6-8-4 \\
(g=0,75) / \text { Vidrio } \\
5+5(g=0,85)\end{array}$ & $3,00 / 5,50$ & \\
\hline $\begin{array}{l}\text { Suelo / planta } \\
\text { baja }\end{array}$ & $\begin{array}{l}\text { Forjado } \\
\text { reticular de } \\
\text { hormigón (PUR } \\
30 \mathrm{~mm} \text { ) }\end{array}$ & 0,68 & 0,49 \\
\hline $\begin{array}{l}\text { Particiones } \\
\text { interiores }\end{array}$ & $\begin{array}{l}\text { Partición de } 12 \\
\mathrm{~cm}\end{array}$ & $2,40 / 2,70$ & 1,2 \\
\hline
\end{tabular}

\section{Criterios de selección}

El edificio fue seleccionado para realizar el estudio por las siguientes razones: es representativo de los edificios construidos en esos años (finales de los 90 , primera década del s. XXI): es un edificio en bloque, con superficies medias, ubicado en un centro urbano y con unas características constructivas convencionales. Fue construido antes de la entrada en vigor de la normativa actual sobre eficiencia energética, como la mayoría de los edificios existentes. Es pues susceptible de rehabilitación. Es de propiedad única, lo que por mediación de los promotores del estudio con la empresa propietaria ha permitido tener acceso directo a datos de consumo de los diferentes suministros, tanto totales como individualizados, siempre manteniendo a salvo la confidencialidad de los datos. El edificio no tiene problemas estructurales, de higiene, de riesgo de exclusión social u otras circunstancias que impidan considerar el caso de estudio como representativo.

\section{Consumo}

El consumo real de gas natural $\left(106,11 \mathrm{kWh} / \mathrm{m}^{2}\right.$ año) y de energía eléctrica $\left(33,1 \mathrm{kWh} / \mathrm{m}^{2}\right.$ útil de vivienda y año) del edificio es ligeramente más bajo (hasta un $10 \%$ ) que los valores medios de un edificio similar en la misma zona climática, según Calener VyP (Ministerio de Vivienda e Instituto para la Diversificación y Ahorro de la Energía -IDAE, 2009) y el Instituto para la Diversificación y Ahorro de la Energía (IDAE (2011). Por lo tanto, se considera que el consumo real se encuentra entre los valores medios, pero variables como los horarios, los hábitos de consumo, el número de habitantes, etc., deben tenerse en cuenta.
Figura 3. Interfaz geométrica Calener VyP para el edificio de viviendas. Fuente: Elaboración Propia, 2012.

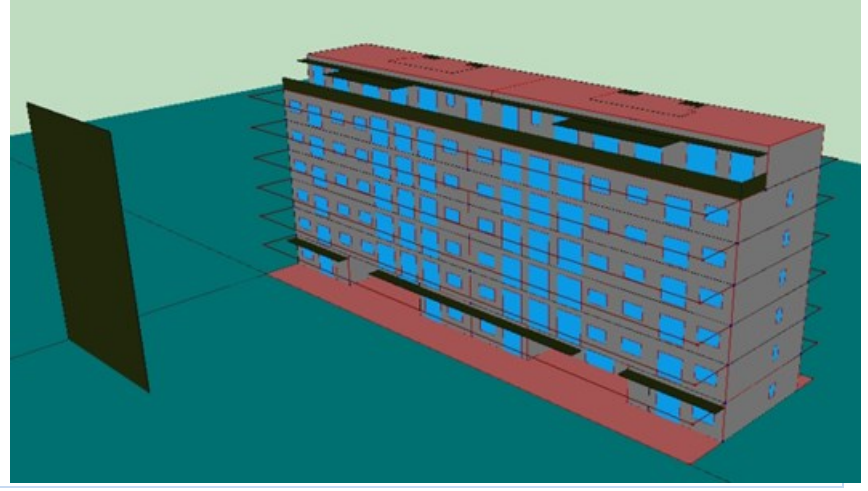

Figura 4. Escala de calificación energética de edificios. Fuente: Calener VYP, 2012.

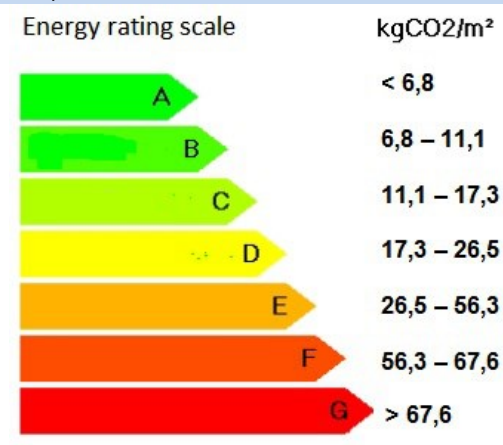

\section{Calificación energética del edificio.}

Los procedimientos simplificados para la calificación energética de edificios existentes CE3 o CE3X, que son los más utilizados en la práctica, desarrollan la metodología de cálculo de una manera indirecta, resultando más conservadores y ofreciendo como resultado una clase de eficiencia energética siempre igual o inferior a la obtenida con Calener VYP que, como ya se ha indicado, es el programa oficial para establecer la calificación energética de viviendas y pequeños edificios del sector terciario en España. Esta circunstancia, unida al hecho de que la utilización de Calener VYP ha permitido asimismo la comparación de los resultados de esta fase del estudio con los resultados de las fases anteriores, animó al equipo investigador a utilizar el programa oficial en lugar de los más sencillos procedimientos simplificados.

Las entradas del programa Calener VYP incluyen la geometría, orientación, sombreamiento, etc.; las características térmicas de la envolvente del edificio (transmisión térmica $U$, puentes térmicos, ventilación, etc.); y las características de las instalaciones de calefacción, refrigeración y agua caliente sanitaria (capacidad nominal, el rendimiento energético, el flujo de aire, etc.) (Figura 3). Calener VyP genera una etiqueta con la calificación energética de los edificios que va desde la $A$, para los edificios más eficientes, a la G (Figura 4). La calificación se establece basándose en las emisiones de $\mathrm{CO}_{2}\left(\mathrm{kgCO}_{2} / \mathrm{m}^{2} \mathrm{año}\right)$ pero el programa también proporciona los resultados de consumo de energía primaria y final. De acuerdo con esta herramienta, la calificación energética del edificio objeto de estudio, en su estado actual, es de $29,4 \mathrm{kgCO} 2 / \mathrm{m}^{2}$ año, lo que equivale a una calificación 


\section{Análisis y selección de las medidas pasivas a aplicar en el edificio}

La eficiencia energética de un edificio se incrementa mediante la mejora de su envolvente y la consiguiente reducción de su demanda de energía, con la sustitución de los sistemas energéticos por unos más eficientes, y con el uso de energías renovables. Las de mejora de la envolvente son casi siempre medidas pasivas, pues consiguen reducción de la demanda como consecuencia de sus propias características geométricas y dimensionales. Pueden aplicarse para calentar o refrigerar el edificio, como sigue:

Estrategias para reducir la demanda de calefacción: mejora de las características térmicas de los cierres opacos (fachadas, cubiertas y suelos); mejora térmica de las ventanas (valores de transmitancia y factor solar, posición respecto a la fachada); incremento de la inercia térmica (cubiertas verdes, aislamientos externos); uso de la energía solar pasiva desde octubre a abril, para la captación solar directa; y reducción de puentes térmicos e infiltraciones de aire.

Estrategias para reducir la demanda de refrigeración: evitar la luz solar en la envolvente del edificio (protecciones solares, fachada ventilada); elementos de sombra que permitan la captación solar en los meses de otoño-invierno (de octubre a abril) y la protección en los meses de primavera-verano (de mayo a septiembre); aumentar la inercia térmica para mantener la temperatura de la noche durante el día en los meses cálidos (cubiertas verdes, aislamiento externo); favorecer la ventilación cruzada y la ventilación por la noche en los meses de junio a septiembre.

De entre las medidas pasivas a aplicar en una hipotética rehabilitación del edificio, en base a las cartas bioclimáticas de Olgyay (1998) y Givoni (1998), y sobre todo considerando las características constructivas y arquitectónicas propias del edificio, se han seleccionado las siguientes: - Rehabilitación energética de las fachadas: con sistemas de aislamiento térmicos exterior tipo SATE (1.1) y (1.2), con fachadas ventiladas (1.3), con aislamiento interior de las paredes exteriores (1.4). Rehabilitación energética de los techos: con aislamiento exterior en cubiertas invertidas ( 2.1 y 2.2$)$, con cubierta verde (2.3), con techos aislados por el interior (2.4). Rehabilitación energética de la planta baja y los pisos exteriores con techos aislados (3). Rehabilitación energética de las ventanas y las puertas de vidrio. En un edificio existente las directrices están definidas y las medidas limitadas, pero es posible modificar las características térmicas de los vacios y añadir sombreado para reducir la demanda de energía en verano con las soluciones siguientes: reemplazando el vidrio existente por uno de baja emisividad (4.1); sustituyendo las ventanas existentes (4.2), (4.3) y (4.4); colocando una nueva ventana (a modo de doble ventana) por el exterior (4.5) y (4.6). Protección del sol en las fachadas este y oeste: a través de lamas o toldos de diferentes modelos (5.1) a (5.4).

Cada una de las medidas para mejora de la envolvente se define, presupuesta y evalúa con Calener VyP, aplicándolas al edificio de forma independiente para así establecer su capacidad para reducir la demanda de energía y las emisiones de $\mathrm{CO}_{2}$. Un último estudio de las soluciones se lleva a cabo mediante la comparación de los resultados de todas las medidas, teniendo en cuenta la relación entre el ahorro de energía final y el coste de cada intervención, como se muestra en la Figura 5.

Figura 5. Relación entre el coste y el ahorro de energía en las medidas de mejora de la envolvente. Eje de ordenadas: coste de cada medida en euros. Eje de abscisas: ahorro energético conseguido en $\mathrm{kWh} / \mathrm{m}^{2}$ construido. Fuente: Elaboración Propia, 2012.

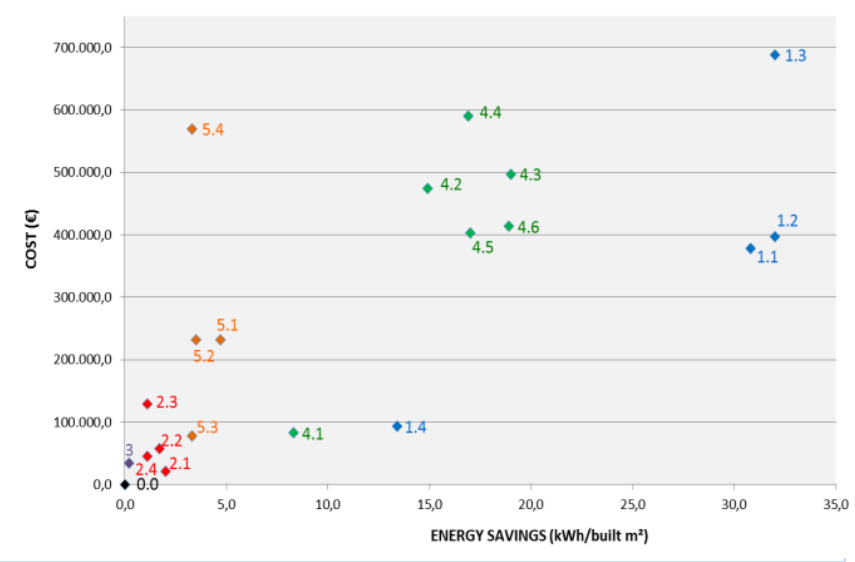

Conocidos estos datos, se han seleccionado aquellas que presentan una mejor relación entre la energía ahorrada ( $k W h)$ y el coste de la intervención, para definir dos envolventes hipotéticas, (E1) y (E2). El objetivo del primer caso de envolvente (E1) era cumplir con la normativa española de eficiencia energética vigente en la fecha del estudio (2012). Con esta envolvente $\mathrm{E} 1$, el edificio reduciría sus emisiones anuales de dióxido de carbono a $16,20 \mathrm{kgCO}_{2} / \mathrm{m}^{2}$ y su demanda total de energía (calefacción, refrigeración y agua caliente sanitaria) sería de de $81,5 \mathrm{kWh} / \mathrm{m}^{2}$ al año. Concretamente, la envolvente E1 incorpora las medidas siguientes: Aumento del aislamiento de las cubiertas y las terrazas de la planta ático con paneles de poliestireno extruido hasta llegar a $80 \mathrm{~mm}$ de espesor. Mejora de la transmitancia de las fachadas que no cumplen con las exigencias del CTE: aislamiento de la fachada de planta baja en contacto con espacios comunes con un trasdosado interior autoportante con $45 \mathrm{~mm}$ de lana mineral; aislamiento de los cerramientos de los tendederos de la fachada oeste mediante un SATE con $30 \mathrm{~mm}$ de poliestireno extruido; y aislamiento de los pilares en esquina con un trasdosado interior.

Aislamiento de las particiones interiores que separan las viviendas de las zonas comunes no calefactadas con un trasdosado autoportante con $45 \mathrm{~mm}$ de lana mineral. Aislamiento del forjado de la planta primera en contacto con el exterior mediante un techo suspendido, con $50 \mathrm{~mm}$ lana mineral en su interior. Aislamiento del forjado de planta baja en contacto con los garajes y trasteros mediante un techo suspendido con aislamiento interior de lana mineral de $30 \mathrm{~mm}$ de espesor. Incorporación de una ventana exterior a las existentes, con un marco de PVC de dos cámaras $\left(2,2 \mathrm{~W} / \mathrm{m}^{2} \mathrm{~K}\right)$ y un vidrio bajo emisivo 4-8-4 $\left(3 \mathrm{~W} / \mathrm{m}^{2} \mathrm{~K}, \mathrm{~g}=0,75\right)$. En los casos en los que no sea posible colocar una nueva carpintería exterior (miradores, puerta de acceso al portal) se sustituye la carpintería existente por otra con marco de PVC de dos cámaras $\left(2,2 \mathrm{~W} / \mathrm{m}^{2} \mathrm{~K}\right)$ y un vidrio bajo emisivo 4-8-4 $(2,3$ $\left.\mathrm{W} / \mathrm{m}^{2} \mathrm{~K}, \mathrm{~g}=0,70\right)$.

Con la segunda envolvente (E2), mejor que la anterior en prestaciones térmicas, el edificio reduciría sus emisiones anuales de dióxido de carbono a $14,90 \mathrm{kgCO}_{2} / \mathrm{m}^{2}$ y su demanda total de energía (calefacción, refrigeración y agua caliente 
sanitaria) sería de 52,8 $\mathrm{kWh} / \mathrm{m}^{2}$ al año. La envolvente E2 incorpora las medidas siguientes: Aumento del aislamiento de las cubiertas y las terrazas de la planta ático con paneles de poliestireno extruido hasta llegar a $120 \mathrm{~mm}$ de espesor. Aislamiento de las fachadas mediante un SATE con $80 \mathrm{~mm}$ de poliestireno extruido, excepto las de los tendederos de la fachada oeste que se aíslan con $60 \mathrm{~mm}$ de poliestireno extruido. Mejora de la transmitancia de las particiones interiores que separan las viviendas y las zonas comunes no calefactadas con un trasdosado autoportante con $45 \mathrm{~mm}$ de lana mineral. Mejora de la transmitancia del suelo de la planta primera en contacto con el exterior mediante un techo suspendido, con $80 \mathrm{~mm}$ lana mineral en su interior. Mejora de la transmitancia del forjado de planta baja en contacto con el espacio no habitable del sótano mediante un techo suspendido, con aislamiento interior de lana mineral de $80 \mathrm{~mm}$ de espesor. Incorporación de una ventana exterior a las existentes, con un marco de PVC de tres cámaras $\left(1,8 \mathrm{~W} / \mathrm{m}^{2} \mathrm{~K}\right)$ y un vidrio bajo emisivo 4-16-4 (1,4 W/m² $\mathrm{K}, \mathrm{g}=0,59)$. En los casos en los que no sea posible colocar una nueva carpintería exterior (miradores, puerta de acceso al portal) sustituir la carpintería existente por otra con marco de PVC de tres cámaras $\left(1,8 \mathrm{~W} / \mathrm{m}^{2} \mathrm{~K}\right)$ y un vidrio bajo emisivo $4-16-4\left(1,4 \mathrm{~W} / \mathrm{m}^{2} \mathrm{~K}, \mathrm{~g}=0,59\right)$.

Tabla 2. Transmitancias de las envolventes E1 y E2. Fuente: elaboración propia, 2013.

\begin{tabular}{|c|c|c|}
\hline $\begin{array}{l}\text { Elemento de } \\
\text { construcción }\end{array}$ & $\begin{array}{l}E 1 \\
U\left(W / m^{2} K\right)\end{array}$ & $\begin{array}{l}\mathrm{E2} \\
\mathrm{U}\left(\mathrm{W} / \mathrm{m}^{2} \mathrm{~K}\right)\end{array}$ \\
\hline Techos & 0,34 & 0,24 \\
\hline Fachada principal & 0,55 & 0,24 \\
\hline Fachada secundaria & 0,80 & 0,37 \\
\hline Marco de ventana & 2,20 & 1,80 \\
\hline Vidrio & 3,00 & 1,40 \\
\hline $\begin{array}{l}\text { Estructura suelo exterior } \\
\text { / Estructura planta baja }\end{array}$ & 0,40 & 0,26 \\
\hline Particiones interiores & 0,68 & 0,53 \\
\hline
\end{tabular}

Tabla 3. Calificaciones energéticas, demandas y costes de la envolvente existente (EO) y de las hipótesis de la envolvente básica (E1) y mejorada (E2). Fuente: elaboración propia, 2013.

\begin{tabular}{llll}
\hline & E0 & E1 & E2 \\
\hline Clase energética $\left(\mathrm{kgCO} / \mathrm{m}^{2}\right)$ & $29,4 \mathrm{E}$ & $23,2 \mathrm{D}$ & $14,5 \mathrm{C}$ \\
\hline Demanda calefacción $\left(\mathrm{kWh} / \mathrm{m}^{2}\right)$ & 73,3 & 49,2 & 31,1 \\
\hline $\begin{array}{l}\text { Variaciones de demanda de } \\
\text { calefacción }\left(\mathrm{kWh} / \mathrm{m}^{2}\right)\end{array}$ & $0,0 \%$ & $32,9 \%$ & $57,6 \%$ \\
\hline Demanda refrigeración $\left(\mathrm{kWh} / \mathrm{m}^{2}\right)$ & 13,1 & 14,4 & 3,8 \\
\hline $\begin{array}{l}\text { Variaciones de demanda de } \\
\text { refrigeración }\left(\mathrm{kWh} / \mathrm{m}^{2}\right)\end{array}$ & 0,0 & $9,9 \%$ & $71,0 \%$ \\
\hline Coste $\left(€ / \mathrm{m}^{2}\right.$ edificio construido) & 0,0 & 71,1 & 132,9 \\
\hline
\end{tabular}

Las transmitancias resultantes de ambas envolventes se detallan en la Tabla 2. En la Tabla 3 se detallan las calificaciones energéticas resultantes como consecuencia de la modificación exclusiva de la envolvente, básica (E1) y mejorada (E2), así como demandas y costes constructivos, en comparación con la envolvente actual del edificio (EO):

\section{Medidas activas seleccionadas}

La mejora de la eficiencia de los sistemas energéticos se logra de forma directa mediante la sustitución de las instalaciones existentes por otras más eficientes y el uso de energías renovables. En función de las características constructivas y arquitectónicas del edifico, su emplazamiento y ubicación, y de los sistemas más comunes utilizados en edificios residenciales, se han seleccionado de común acuerdo con los promotores del estudio trece sistemas energéticos (ver Tabla 4), que han sido evaluados, calculados y presupuestados. Entre ellos se han incluido como casos de uso de energías renovables, sistemas de agua caliente sanitaria con paneles de energía solar térmica, y calderas de biomasa. Se han considerado sistemas de calefacción, refrigeración y ACS en dos bloques: a) Sistemas de calefacción y agua caliente sanitaria: Han sido analizados 3 sistemas individuales y 4 centralizados. Incluyen la instalación de calderas de condensación individual y colectiva, una caldera de biomasa, tecnologías de microgeneración, un sistema solar térmico que cubre el $70,2 \%$ de las necesidades de agua caliente sanitaria y calefacción por suelo radiante. B) Sistemas de calefacción, refrigeración y de agua caliente sanitaria: Han sido analizados 2 sistemas individuales y 4 centralizados. Estos sistemas, además de lo anterior, incluyen equipos de expansión directa y de conductos, enfriadores, bombas de calor eléctricas, bombas de calor de gas natural, y calefacción y refrigeración por suelo radiante.

Tabla 4. Relación de sistemas energéticos considerados en el estudio. Fuente: elaboración propia, 2013.

\begin{tabular}{ll}
\hline SISTEMA & DESCRIPCIÓN \\
\hline S1 & Calefacción y ACS \\
\hline S2 & $\begin{array}{l}\text { Sistema con caldera estándar de gas natural, con acumulador } \\
\text { integrado y radiadores de aluminio (edificio existente). }\end{array}$ \\
\hline S3 & $\begin{array}{l}\text { Sistema con calderas de condensación de gas natural y } \\
\text { radiadores de aluminio. 70\% de la demanda de ACS cubierta } \\
\text { por energía solar térmica. }\end{array}$ \\
\hline S4 & $\begin{array}{l}\text { Sistema con calderas de condensación de gas natural y la } \\
\text { calefacción por suelo radiante. 70\% de la demanda de ACS } \\
\text { cubierta por energía solar térmica. }\end{array}$ \\
\hline S5 & $\begin{array}{l}\text { Sistema con calderas de condensación de gas natural y } \\
\text { radiadores de aluminio. 70\% de la demanda de ACS cubierta } \\
\text { por energía solar térmica. }\end{array}$ \\
\hline S6 & $\begin{array}{l}\text { Sistema con calderas de condensación de gas natural y suelo } \\
\text { radiante. 70\% de la demanda de ACS cubierta por energía } \\
\text { solar térmica. }\end{array}$ \\
\hline S7 & $\begin{array}{l}\text { Sistema colectivo con calderas de condensación de gas } \\
\text { natural y la calefacción por suelo radiante. La demanda de } \\
\text { ACS cubierta por un sistema de micro cogeneración }\end{array}$ \\
\hline S12 & Sistema con calderas de biomasa y suelo radiante. \\
\hline Calefacción, refrigeración y ACS \\
\hline S10
\end{tabular}




\section{Combinación de medidas pasivas y activas. Resultados}

La rehabilitación energética necesita de combinación de medidas pasivas como paso previo a la aplicación de medidas activas. En este caso de estudio, los 13 sistemas energéticos (medidas activas) aplicados en el edificio configurado con las 2 hipótesis de envolventes E1 y E2 (medidas pasivas) producen 26 escenarios que son evaluados con Calener VyP y valorados económicamente. Las hipótesis se nombran primero con el tipo de envolvente (E1 o E2) y posteriormente con la denominación del sistema energético (S1 a S13). La Figura 6 expresa la relación entre el coste absoluto en euros de las mejoras introducidas y el ahorro de energía ( $\mathrm{kWh} / \mathrm{m}^{2}$ año) en los 13 escenarios realizados con la envolvente E1. En el eje de ordenadas se representa el coste absoluto (en euros) y en el eje de abscisas el ahorro de energía (en $\mathrm{kWh} / \mathrm{m}^{2}$ año). La Figura 7 expresa la relación entre el coste absoluto en euros de las mejoras introducidas y el ahorro de energía ( $\mathrm{kWh} / \mathrm{m}^{2}$ año) en los 13 escenarios realizados con la envolvente E2. En el eje de ordenadas se representa el coste absoluto (en euros), y en el eje de abscisas el ahorro de energía (en $\mathrm{kWh} / \mathrm{m}^{2}$ año).

Figura 6. En el eje de ordenadas, el coste en euros de las mejoras introducidas sobre las hipótesis para la envolvente E1, y en el eje de abscisas el correspondiente ahorro de energía en $\mathrm{kWh} / \mathrm{m}^{2}$ año. Fuente: Elaboración Propia, 2012.

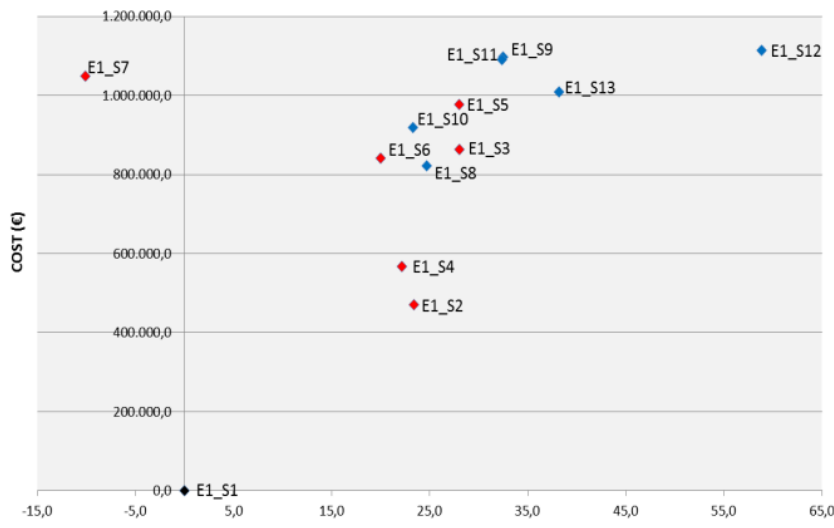

ENERGY SAVINGS (kWh/built $\left.\mathrm{m}^{2}\right)$

Figura 7. En el eje de ordenadas, el coste en euros de las mejoras introducidas sobre las hipótesis para la envolvente E2, y en el eje de abscisas el correspondiente ahorro de energía en $\mathrm{kWh} / \mathrm{m}^{2}$ año. Fuente: Elaboración Propia, 2012.

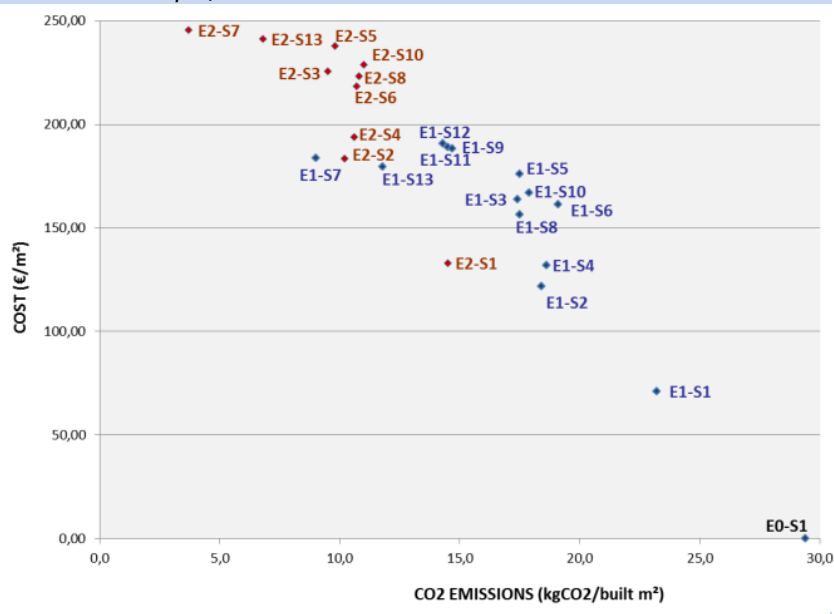

La Figura 8 ilustra la relación entre el coste por metro cuadrado (en euros) de las mejoras introducidas y las emisiones de $\mathrm{CO}_{2}$ $\left(\mathrm{kgCO}_{2} / \mathrm{m}^{2}\right.$ año) estimadas para cada uno de los 26 escenarios realizados con ambas envolventes E1 y E2. Se parte de la situación original, EO-S1, que es la del edificio tal como está en la actualidad. En el eje de ordenadas se sitúa el coste por metro cuadrado (en euros) y en el eje de abscisas se sitúan las emisiones de $\mathrm{CO}_{2}$ estimadas para cada escenario (en $\mathrm{kgCO}_{2} / \mathrm{m}^{2}$ año).

La Figura 9 expresa la relación entre el coste por metro cuadrado (en euros) de las mejoras introducidas en cada una de las 26 hipótesis analizadas, y el ahorro de energía (en $\mathrm{kWh} / \mathrm{m}^{2}$ construido) estimado para cada una de ellas. Se parte de la situación original, E0-S1, que es la del edificio tal como está en la actualidad. En el eje de ordenadas se sitúa el coste por metro cuadrado (en euros) y en el eje de abscisas se sitúa el ahorro de energía estimado (en $\mathrm{kWh} / \mathrm{m}^{2}$ y año).

Figura 8. Ordenadas: coste de cada uno de los 26 escenarios de rehabilitación (en euros $/ \mathrm{m}^{2}$ ). Abscisas: emisiones de $\mathrm{CO}_{2}$ estimadas para cada escenario (en $\mathrm{kgCO}_{2} / \mathrm{m}^{2}$ año). Fuente: Elaboración Propia, 2012.

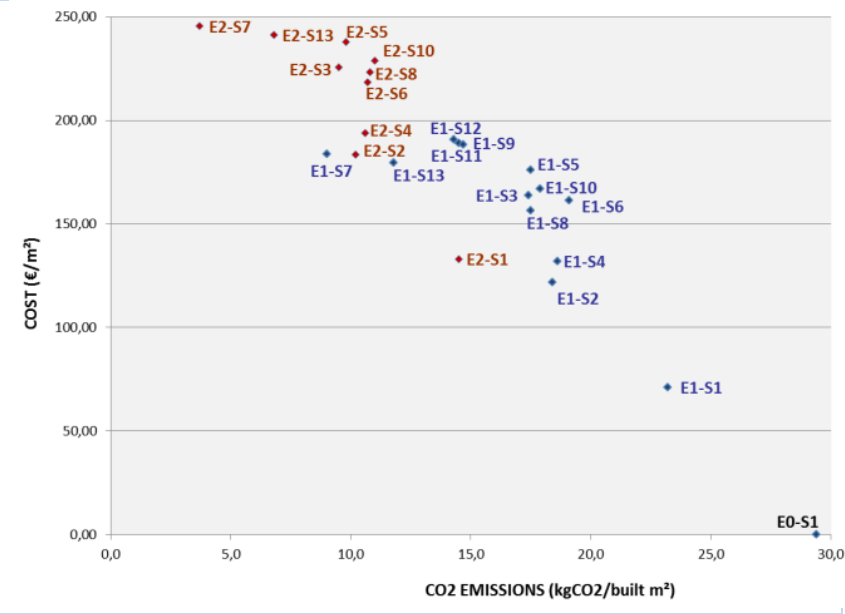

Figura 9. Ordenadas: coste por metro cuadrado en euros de cada una de las 26 hipótesis analizadas. Abscisas: ahorro de energía correspondiente estimado en $\mathrm{kWh} / \mathrm{m}^{2}$ y año. Fuente: Elaboración Propia, 2012.

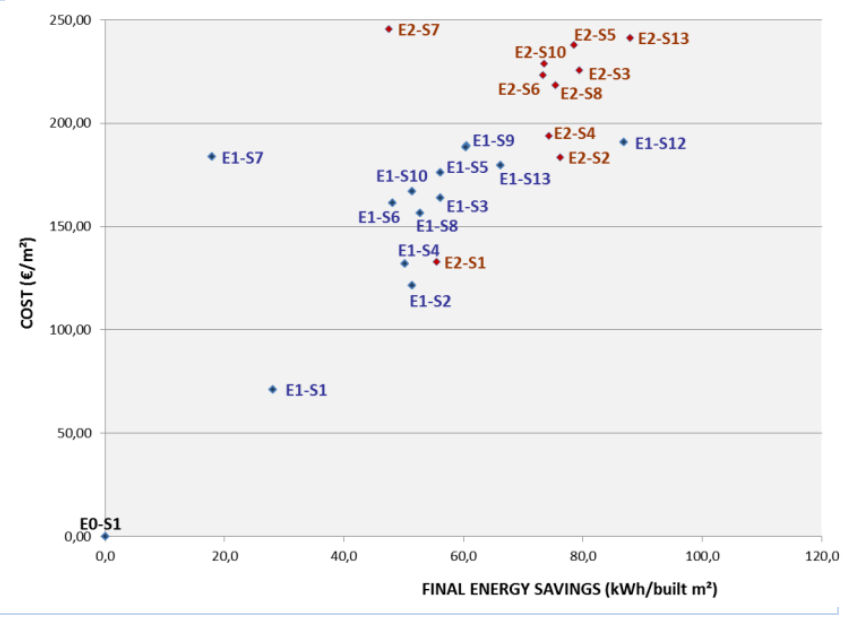


A los resultados reseñados en las figuras 6 a 9 se añade otro factor más de estudio, este relacionado con los precios de los combustibles en España en la fecha de realización del trabajo (2012). Se considera así otro aspecto importante, como es el coste económico que tiene la rehabilitación energética para cada usuario o consumidor, y la repercusión del ahorro en los consumos energéticos, sin tener en cuenta otros elementos de oportunidad como son el término fijo, alquileres e impuestos de la tarifa energética. La Tabla 5 presenta las calificaciones energéticas obtenidas mediante las 26 hipótesis y su relación con el coste por metro cuadrado construido y en valores absolutos para cada vivienda de dos dormitorios; y los ahorros, tanto en consumo energético como en la factura energética, para el consumidor.

\section{Discusión de resultados y comparativa con experiencias similares en otros países}

Los resultados muestran las posibilidades y el potencial beneficio derivado de la reducción la demanda de energía en el parque construido de viviendas, y por tanto de reducir las emisiones de $\mathrm{CO}_{2}$, mediante una selección de determinadas medidas para obtener la mejor relación entre inversión y resultados. El caso de estudio se refiere a un edificio concreto, pero que tiene unas características muy generalizables. $Y$ se demuestra que cualquier inversión debe ser cuidadosamente analizada para obtener los mejores resultados.

Pero por otra parte también demuestra la dificultad de que las operaciones de rehabilitación energética sean financiables por sí mismas. El enfoque de la rehabilitación energética debe integrarse en un punto de vista mucho más amplio que el de la energía, para incardinarse en el ámbito de la sostenibilidad, es decir, contemplando al mismo tiempo aspectos sociales y económicos, como bien demuestran herramientas de ayuda a la toma compleja de decisiones como EPIQR (Genre et al. 2000), u otras investigaciones sobre casos de estudio similares (Konstantinou y Knaack, 2013). En lo que respecta a los aspectos económicos, la financiación y el apoyo estatal es uno de los puntos clave. Varios países de la Unión Europea así lo han entendido, desarrollando mecanismos, programas $y$ políticas financieras apoyadas por bancos o entidades privadas. Es el caso de las Empresas de Servicios Energéticos (ESEs), que ofrecen servicios de rehabilitación energética sin ningún coste por adelantado, y recuperan los pagos a través del ahorro en las facturas energéticas. Estos sistemas u otros sistemas parecidos, como el programa Green Deal del Reino Unido, el programa alemán del banco estatal KfW (con tasas de interés igual o por debajo del 2,75\%), el programa PACE (Property Assessed Clean Energy programme) de Estados Unidos, etc., establecen medidas de incentivos económicos. En España se han establecido ya ayudas a la rehabilitación desde los ámbitos estatal y autonómico que están activas desde octubre de 2013 (Secretaría de Estado de Energía, 2013).

El éxito de la rehabilitación necesita asimismo del incentivo del ahorro de energía para el usuario, y por tanto de campañas de información, publicidad y refuerzo, incluido el uso prestigioso de una marca o logo reconocido. Esta ha sido una de las estrategias seguidas por ENERGY STAR, que mediante el programa HPwES -Home Performance with Energy Star(HPwES, 2013), ha mejorado desde el año 2002 más de 275.000 viviendas en Estados Unidos. Más concretamente, el programa NYSERDA ha mejorado más de 11.000 viviendas, ahorrando más de 16.000 MWh de electricidad y $700.000 \mathrm{MMBtu}$ de combustibles fósiles (Tonn et al. 2013). En lo referente a ahorros por cada vivienda, el programa de Austin (Texas) de 2007 consiguió ahorros en refrigeración del 25-35\%, valorados entre 460 y 640 dólares anuales. El programa en Wisconsin, consiguió unas reducciones medias por vivienda de aproximadamente $1,6 \mathrm{~kW}$ en verano y de $0,9 \mathrm{~kW}$ en invierno.

Así pues, los resultados alcanzados en otros países (Liu y Guo, 2013) presentan bastantes similitudes con el estudio realizado y con otros semejantes realizados en España. Por ejemplo el de WWF (WWF, 2012), que concluye que la demanda de energía puede reducirse en un intervalo del $66-83 \%$ con una mejora cuidadosa del aislamiento. Considerando el precio medio de $0,076 € / K W h$ en concepto de energía, el informe de WWF estima que el ahorro energético anual derivado de diversas intervenciones de aislamiento representa entre 450-680 euros para un hogar medio de $81 \mathrm{~m}^{2}$, y concluye que la recuperación de la inversión por intervenciones de aislamiento se establece en unos 10-11 años. La incorporación de producción de agua caliente sanitaria con energía solar térmica y de electricidad con energía solar fotovoltaica, con una actualización de los equipos de aire acondicionado y de calderas, puede conseguir una reducción del consumo energético de hasta el $85 \%$ y una disminución de las emisiones de $\mathrm{CO}_{2}$ del $82 \%$ con respecto a un escenario que mantuviera hipotéticamente la tendencia actual. La amortización de una rehabilitación profunda de este tipo se sitúa en unos 29 años. Por ello es importante realizar trabajos específicos analizando casos de estudio como el del presente trabajo, que muestra que la mejor opción en la relación inversión/resultados es la de obtener reducciones de energía del 50\% con una inversión moderada (alrededor de $214 € / \mathrm{m}^{2}$ ). Otras posibilidades de obtener mayores ahorros, de hasta un $85 \%$, requieren una inversión mucho mayor (alrededor de 301 $€ / \mathrm{m}^{2}$ de vivienda) que costará más tiempo amortizar.

Tabla 5. Calificaciones energéticas obtenidas con las 26 hipótesis. Costes por metro cuadrado construido y en valor absoluto para cada vivienda de dos dormitorios. Ahorro de consumo energético y de factura energética para el consumidor. Fuente: elaboración propia, 2013.

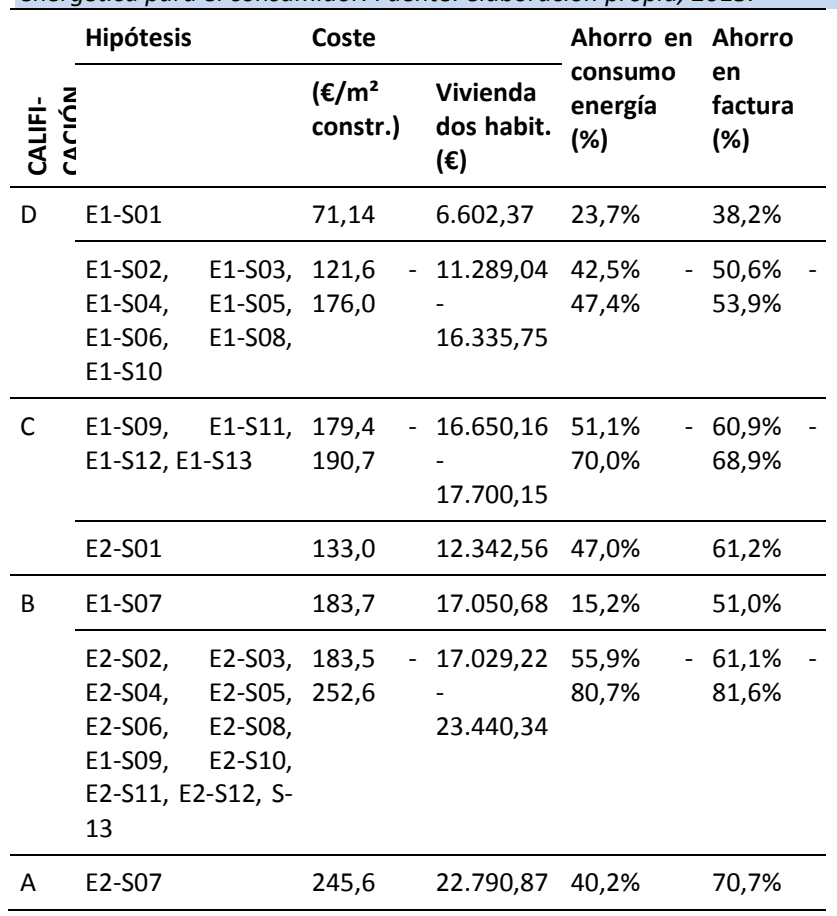


Otro de los resultados de especial interés es la necesidad de considerar la rehabilitación en términos de mantenimiento y mejora del patrimonio construido, de confort y bienestar para los usuarios, y de mitigación del cambio climático. Todas estas razones están además impregnadas de motivaciones éticas, puesto que las razones que fundamentan la rehabilitación energética proceden de diversos campos. Trebilcock establece que las políticas estatales coinciden con la percepción que del problema tienen los arquitectos de los respectivos países (Trebilcock, 2011). En los países europeos los arquitectos persiguen principalmente objetivos ambientales globales, concretamente la disminución de las emisiones de $\mathrm{CO}_{2}$ asociadas a las edificaciones para con ello mitigar el calentamiento global. En Chile la motivación principal parece ser la consecución de objetivos sociales, como mejorar las condiciones de habitabilidad de las personas, frente a objetivos ambientales globales como mitigar el cambio climático. Las motivaciones éticas forman parte del conjunto de razones que estimulan acciones de este tipo, dictadas desde la ética ambiental. En España, el reciente Real Decreto por el que se regula la rehabilitación de viviendas (Ministerio de Fomento, 2013) presenta como objetivo prioritario la garantía constitucional del disfrute de una vivienda digna.

La creación de puestos de trabajo es otra de estas razones. Según WWF, (WWF, 2012) entre 2001 y 2006 la Alianza Alemana para el Trabajo y el Medio Ambiente utilizó políticas y subvenciones públicas para dedicar una inversión total de 15.200 millones de euros en rehabilitación energética de edificios, lo que trajo como consecuencia la creación o mantenimiento de 140.000 puestos de trabajo. El mismo informe estima la creación de 18 puestos directos de trabajo por cada millón de euros invertido en rehabilitación profunda, y un $50 \%$ adicional por actuaciones más simples, lo que en España podría suponer la creación o mantenimiento de cerca de 140-150.000 puestos de trabajo, por lo menos hasta 2044.

\section{Conclusiones}

De las tablas aportadas se pueden extraer muchas y muy diferentes conclusiones, pero probablemente la principal es que el éxito de la rehabilitación energética depende tanto de la inversión económica como de la adecuada selección de las medidas que se implementan. En cualquier caso y concretando, se señalan como más significativas al enfoque aquí planteado las siguientes: Las puntuaciones más altas y un ahorro energético de hasta el $80 \%$ solo se puede conseguir con las inversiones más grandes, de hasta $23.440 €$ para una vivienda de dos dormitorios (77,67 $\mathrm{m}^{2}$ construidos). Pero el ahorro de energía de alrededor del $50 \%$ se consigue con una inversión intermedia, alrededor de $16.650 €$ para la misma vivienda de dos dormitorios. En rehabilitación, la mejora del rendimiento energético del edificio depende de la inversión y de la selección de soluciones apropiadas (protección solar, aislamiento térmico, sistemas de eficiencia energética y energías renovables) mediante un análisis que tenga en cuenta el costebeneficio de la intervención. Para lograr buenas calificaciones energéticas son necesarias tanto la mejora térmica de la envolvente (sistemas pasivos) como la mejora de los sistemas energéticos (sistemas activos). La mejora del aislamiento de las fachadas es la intervención de rehabilitación energética que tiene la mejor relación entre rentabilidad económica, ahorro de energía y reducción de las emisiones de $\mathrm{CO}_{2}$. La mejora de las características térmicas de las ventanas y su protección solar (reduce la demanda de refrigeración hasta un 60\%) produce también un significativo ahorro de energía, pero tiene largos periodos de amortización. La instalación de calderas de condensación y un sistema de energía solar térmica para producción de ACS son las intervenciones activas más rentables. Los sistemas energéticos que presentan una mejor relación entre la rentabilidad, ahorro de energía y reducción de emisiones de $\mathrm{CO}_{2}$ son los que tienen gas o bomba de calor eléctrica con calefacción y refrigeración por suelo radiante.

En cualquier caso, la rehabilitación energética no se justifica solo en términos económicos, aun considerando dentro de estos que la rehabilitación energética puede significar un incremento en el valor de la propiedad, y que la fluctuación de los precios de combustible es imprecisa y presumiblemente al alza. La rehabilitación energética implica la mejora de aspectos del edificio que no son cuantificables, tales como aumento del confort, la mejora del aislamiento acústico, la habitabilidad, la salud o la eliminación, en casos extremos pero más frecuentes de lo imaginable, de situaciones de pobreza energética. Si la rehabilitación energética se hace coincidir con la rehabilitación constructiva de mantenimiento habitual del edificio, la diferencia entre ambas será entonces mínima y mucho más fácil de amortizar.

\section{Agradecimientos.}

Este trabajo es resultado del acuerdo firmado por los promotores del estudio, la Fundación ASPRIMA, GAS NATURAL - FENOSA, URSA URALITA y UPONOR, y el Grupo de Investigación Sostenibilidad en la Construcción e Industria (giSCl), de la Universidad Politécnica de Madrid, para el desarrollo del proyecto de investigación PRECOST\&E REHABILITACIÓN.

\section{Referencias.}

Comisión Europea (2008). COMUNICACIÓN DE LA COMISIÓN AL PARLAMENTO EUROPEO, AL CONSEJO, AL COMITÉ ECONÓMICO Y SOCIAL EUROPEO Y AL COMITÉ DE LAS REGIONES. Dos veces 20 para el 2020. El cambio climático, una oportunidad para Europa. Obtenido de http://eurlex.europa.eu/LexUriserv/LexUriServ.do?uri=COM:2008:0030:FIN:es:PDF__[visitado 29.10.13]

Environmental Protection Agency, EPA (EEUU). HOME PERFORMANCE WITH ENERGY STAR. Obtenido en http://www.energystar.gov/ [visitado 15.02.14]

GARCíA NAVARRO, J.; GONZÁLEZ DíAZ, MJ. \& VALDIVIESO, M. (2010). Estudio PRECOST\&E. Obtenido de http://www.asprima.es/Noticias/Publicaciones/Estudios/Estudio-PRECOSTE/ [visitado 29.10.13]

GARCÍA NAVARRO, J., GONZÁLEZ DíAZ, M.J. \& VALDIVIESO, M. (2014). Estudio PRECOST\&E: Evaluación de los costes constructivos y consumos energéticos derivados de la calificación energética en un edificio de viviendas situado en Madrid. INFORMES DE LA CONSTRUCCIÓN, 66(535). doi: 10.3989/ic.13.052.

GENRE, J.L., FLOURENTZOS, F., STOCKLI, T. (2000). Building refurbishment: habitat upgrading. Energy and Buildings, 31(2), 155-157.

GIVONI, B. (1998). Climate considerations in building and urban design, Van Nostrand Reinhold, New York: John Wiley. 
Instituto para la Diversificación y Ahorro de la Energía, IDAE (2011) Estudio sobre Consumo Energético del Sector Residencial en http://www.idae.es/index.php/id.171/mod.noticias/mem.detalle [visitado 29.10.13]

KONSTANTINOU, T., \& KNAACK, U. (2013). An approach to integrate energy efficiency upgrade into refurbishment design process, applied in two case-study buildings in Northern European climate. Energy and Buildings, 59(2013), 301-309.

LIU, Y., \& GUO, W. (2013), Effects of energy conservation and emission reduction on energy efficiency retrofit for existing residence: $A$ case from China. Energy and Buildings, 61(2013), 61-72.

Ministerio de Fomento de España (2013). Real Decreto 233/2013 Madrid, España.

Ministerio de Vivienda de España e Instituto para la Diversificación y Ahorro de la Energía, IDAE (2009). Calener-VYP v1.0. Manual de usuario. Madrid. Obtenido de http://www.minetur.gob.es/energia/desarrollo/EficienciaEnergetica/CertificacionEnerg etica/DocumentosReconocidos/ProgramaCalener/CalenerVYP1/Manual_de_usuario.pdf [visitado 29.10.13]

OLGYAY, V. (1998). Arquitectura y clima. Manual de diseño bioclimático para arquitectos y urbanistas. Editorial Gustavo Gili, Barcelona.

Parlamento Europeo (2010). DIRECTIVA 2010/31/UE DEL PARLAMENTO EUROPEO Y DEL CONSEJO de 19 de mayo de 2010 relativa a la eficiencia energética de los edificios (refundición). Obtenido de http://eur-

lex.europa.eu/LexUriServ/LexUriServ.do?uri=OJ:L:2010:153:0013:0035:Es:PDF [visitado 29.10.13]

TONN, B., HAWKINS, B., SCHWEITZER, M., EISENBERG, J. (2013). Process evaluation of the home performance with ENERGY STAR Program. Energy Policy, 56 (2013), 371-381.

TREBILCOCK, M. (2011). Percepción de barreras a la incorporación de criterios de eficiencia energética en las edificaciones. Revista de la Construcción, 10(1), 4-14.

WWF/Adena (2010) World Wildlife Fund for Nature. Potencial de ahorro energético y de reducción de emisiones de $\mathrm{CO} 2$ del parque residencial existente en España en 2020. Obtenido en http://awsassets.wwf.es/downloads/informe_potencial_rehab_vf_dic2010.pdf [visitado 29.10.13]

WWF (2012) World Wildlife Fund for Nature. Retos y oportunidades de financiación para la rehabilitación energética de viviendas en España. Obtenido en http://www.wwf.es/?20627/WWF-propone-larehabilitacin-energtica-de-ms-de-tres-millones-de-viviendas-para-reducir-las-emisionesde-CO2_[visitado 17.02.14] 